\title{
Simulations of Heated Bluff-Bodies with the Multi-Speed Entropic Lattice Boltzmann Method
}

\author{
Nicolò Frapolli $^{1}$ - Shyam S. Chikatamarla ${ }^{1}$. \\ Ilya Karlin ${ }^{1}$
}

Received: 6 September 2015 / Accepted: 14 September 2015 / Published online: 24 September 2015

C) Springer Science+Business Media New York 2015

\begin{abstract}
Two-dimensional simulations of heated circular and square cylinders are performed in order to validate a recently introduced entropic thermal lattice Boltzmann model (Frapolli et al. in Phys Rev E 90:043306, 2014), and the new boundary conditions for complex walls and multi-speed lattices. Validation is carried out by comparing simulation results with other reference simulations and experiments. For the hydrodynamic field, the wake structure was analyzed by means of the wake recirculation length for both steady and unsteady twodimensional regimes, by comparing the Strouhal number of the vortex shedding associated with the first instability of the hydrodynamic field, and qualitatively by showing the streamlines for different Reynolds numbers. For the thermal part of the simulations, isotherms and mean Nusselt number are qualitatively and quantitatively compared with the literature results. All the computed quantities are found to agree excellently with the reference data available in the literature. This opens door for accurate simulations in complex geometries in thermal and compressible flow regimes with multi-speed lattices.
\end{abstract}

Keywords Entropic LBM - Thermal LBM - LBM walls boundaries · Multi-speed LBM

\section{Introduction}

Thermo-hydrodynamics phenomena such as heat transfer through forced and natural convection are widely spread in engineering applications, ranging from heat exchangers tubes,

Nicolò Frapolli
frapolli@lav.mavt.ethz.ch
Shyam S. Chikatamarla
chikatamarla@lav.mavt.ethz.ch
Ilya Karlin
karlin@lav.mavt.ethz.ch
$1 \quad$ Department of Mechanical and Process Engineering, ETH Zurich, 8092 Zurich, Switzerland 
chimney stacks, cooling towers, offshore structures and electronic cooling, and has been a subject of interest for several decades. Many experimental and numerical methods have been developed for the study of fluid flow interacting with obstacles which exchange heat and momentum. In this context, the lattice Boltzmann method (LBM) has become, recently, a particularly attractive approach in the simulation of fluid dynamics problems, covering a broad range of applications: turbulent flows [1], micro-flows, porous media, multiphase flows [2], relativistic hydrodynamics and beyond [3]. The LB method solves numerically a set of discrete kinetic equations for the populations $f_{i}(\boldsymbol{x}, t)$, designed to reproduce, in the hydrodynamic limit, the desired set of equations; in case of thermal and compressible flows these are the thermo-hydrodynamic equations, which include the Navier-Stokes and the energy equations. Each population is associated with a discrete velocity $\boldsymbol{c}_{i}$ that fits into a regular spatial lattice with nodes $\boldsymbol{x}$, at time $t$. This formulation enables a simple and highly efficient "stream-along-links-and-equilibrate-at-nodes" realization of the LBM algorithm, which is key to both computational efficiency and numerical accuracy of the scheme. Primary success of the LBM resides in the simulation of incompressible flows on standard lattices with relatively few velocities [4-7]; good performance has been achieved recently with the unconditionally stable entropic lattice Boltzmann method (ELBM) [8], which enables simulation of turbulent incompressible flows at high Reynolds number in complex geometries. Recently, the ELBM has been extended for the simulation of thermal and compressible flows at low Mach numbers by using lattices with more velocities [9].

While the basics of wall boundary conditions treatment for isothermal lattice Boltzmann on standard lattices has been studied long ago [10], there is no clear wall boundary treatment for multispeed lattices. In fact, as it will be explained in more details in Sect. 2.3, not only populations associated to the wall nodes are missing, but also populations residing on the "fluid" nodes are. In order to overcome the problem of the boundary conditions for the wall with multi-speed lattices, two main approaches were proposed in the literature: the kineticdiffuse boundary conditions $[11,12]$ and the so-called "regularized boundary conditions" $[13,14]$. Both approaches, however, are restricted to flat walls, precluding the simulation of complex and curved geometries. With the wall boundary conditions proposed in [9], and used in this work, this problem is overcome. The general idea is based on the Tamm-MottSmith (TMS) boundary conditions [8] and the regularized boundary conditions, but with some important modifications, which are described in Sect. 2.3.

Main objectives of this work are to further validate the multi-speed entropic TLBM in the heat transfer domain and, most importantly, are to extend the new boundary conditions of [9] for complex walls. To this end, a typical situation represented by the flow field around a circular and a rectangular cylinder is simulated in a forced convection regime, for a low to medium range of Reynolds ( $\mathrm{Re}$ ) numbers, in two-dimensions. Many quantitative results of the simulations are compared with other simulations and experiments. First, the hydrodynamic characteristics of the wake for both the geometries are analyzed; compared are the characteristic sizes of the wakes, as well as the vortex shedding frequency. In the second part of the work, the capabilities of the model to reproduce correctly heat transfer phenomena are tested by comparing the Nusselt number $(\mathrm{Nu})$ for a wide range of Reynolds number.

The outline of the paper is as follows: in Sect. 2 the multi-speed entropic TLBM is briefly presented, including detail of the boundary conditions. In Sect. 3, details concerning the implementation of the model are listed, together with the information about the numerical setup. In Sect. 4 the results obtained with the LBM are compared with various numerical and experimental data. In Sect. 5 we draw the conclusions. 


\section{The Multi-Speed Entropic Thermal Lattice Boltzmann Model}

\subsection{Lattice Boltzmann Kinetic Equation}

In the entropic thermal LBM, we solve a kinetic equation for the populations $f_{i}(\boldsymbol{x}, t)$, corresponding to discrete velocities $\boldsymbol{c}_{\boldsymbol{i}}$ at position $\boldsymbol{x}$ and time $t$; the generalized LB kinetic equation can be written as:

$$
f_{i}\left(\boldsymbol{x}+\boldsymbol{c}_{i} \delta t, t+\delta t\right)-f_{i}(\boldsymbol{x}, t)=Q_{i},
$$

where $Q_{i}$ is the collision integral. In particular, for the lattice Bhatnagar-Gross-Krook (LBGK) model, the collision integral depends only on one relaxation parameter $\omega_{1}$ :

$$
Q_{i}=\omega_{1}\left(f_{i}^{e q}-f_{i}\right),
$$

where $f_{i}^{e q}$ is the local equilibrium distribution, which satisfies the local conservation laws of a $D$-dimensional ideal gas:

$$
\begin{gathered}
\rho=\sum_{i=1}^{Q} f_{i}^{e q}, \quad \rho u_{\alpha}=\sum_{i=1}^{Q} f_{i}^{e q} c_{i \alpha}, \\
2 \rho E=\sum_{i=1}^{Q} f_{i}^{e q} c_{i \alpha} c_{i \alpha}=D \rho T+\rho u_{\alpha} u_{\alpha},
\end{gathered}
$$

where $D$ is the space dimension. However, when simulating thermal flows, a more advanced model for the collision integral is needed, in order to address the variable Prandtl number $\operatorname{Pr}=$ $\frac{\mathrm{c}_{\mathrm{p}} \mu}{\kappa}$, and then to vary the viscosity $\mu$ and the thermal conductivity $\kappa$ independently. One way to compute the collision integral for such cases is based on the the quasi-equilibrium model presented in [9], where one additional relaxation parameter, $\omega_{2}$, and a quasi-equilibrium state $f_{i}^{*}$ are included; the discretized kinetic equation for the quasi-equilibrium model becomes:

$$
f_{i}\left(\boldsymbol{x}+\boldsymbol{c}_{i} \delta t, t+\delta t\right)-f_{i}(\boldsymbol{x}, t)=\omega_{1}\left(f_{i}^{*}-f_{i}\right)+\omega_{2}\left(f_{i}^{e q}-f_{i}^{*}\right) .
$$

The quasi-equilibrium state $f_{i}^{*}$, in addition to the conserved quantities, is designed to conserve also the prescribed "slow variables", or "quasi-conserved quantities", which are chosen depending on the the Prandtl number. For $\operatorname{Pr}<\operatorname{Pr}_{\mathrm{BGK}}$, where $\operatorname{Pr}_{\mathrm{BGK}}=1$, the centered heat flux tensor

$$
\underline{Q}_{\alpha \beta \gamma}=\sum_{i=1}^{Q} f_{i}^{*}\left(c_{i \alpha}-u_{\alpha}\right)\left(c_{i \beta}-u_{\beta}\right)\left(c_{i \gamma}-u_{\gamma}\right),
$$

is conserved by the right hand side of (4), while for $\operatorname{Pr}>\operatorname{Pr}_{\mathrm{BGK}}$, it is the centered stress tensor

$$
\underline{P}_{\alpha \beta}=\sum_{i=1}^{Q} f_{i}^{*}\left[\left(c_{i \alpha}-u_{\alpha}\right)\left(c_{i \beta}-u_{\beta}\right)-\frac{2}{D} \delta_{\alpha \beta}\left(\boldsymbol{c}_{i}-\boldsymbol{u}\right)^{2}\right],
$$

that needs to be conserved. 


\subsection{Model Description}

The discrete velocity set is chosen based on two criteria: the first is that a sufficient number of discrete velocities must be retained, since it is required to ensure enough symmetry of the lattice to recover the Fourier-Navier-Stokes equations in the low Mach number limit. The second criterium is that the lattice must support entropy, and that it can be derived from the theory of admissible higher-order lattices [15]. In this paper, the model and the lattice of [9] are used; the velocity set is composed of the following six energy shells:

$$
\begin{aligned}
& C_{0}=\{(0,0)\}, \\
& C_{1}=\{(0, \pm 1),( \pm 1,0)\}, \\
& C_{2}=\{( \pm 1, \pm 1)\}, \\
& C_{3}=\{(0, \pm 3),( \pm 3,0)\}, \\
& C_{4}=\{( \pm 1, \pm 3),( \pm 3, \pm 1)\}, \\
& C_{5}=\{( \pm 3, \pm 3)\},
\end{aligned}
$$

which gives a total of 25 velocities for $D=2$. The equilibrium and quasi-equilibrium populations can be written in a compact form, up to third order in the velocity, as:

$$
\begin{aligned}
F_{i}= & W_{i}\left(\rho+\frac{M_{\alpha} c_{i \alpha}}{T}+\frac{\left(M_{\alpha \beta}-\rho T \delta_{\alpha \beta}\right) X_{i, \alpha \beta}}{2 T^{2}}\right) \\
& +W_{i}\left(\frac{\left(M_{\alpha \beta \gamma}-\rho T\left(u_{\alpha} \delta_{\beta \gamma}+u_{\beta} \delta_{\alpha \gamma}+u_{\gamma} \delta_{\alpha \beta}\right)\right) Y_{i, \alpha \beta \gamma}}{6 T^{3}}\right) .
\end{aligned}
$$

where

$$
\begin{gathered}
X_{i, \alpha \beta}=c_{i \alpha} c_{i \beta}-T \delta_{\alpha \beta}, \\
Y_{i, \alpha \beta \gamma}=c_{i \alpha} c_{i \beta} c_{i \gamma}-T\left(c_{i \gamma} \delta_{\alpha \beta}+c_{i \beta} \delta_{\alpha \gamma}+c_{i \alpha} \delta_{\beta \gamma}\right),
\end{gathered}
$$

and where the weights $W_{i}(T)$ depends on the temperature and read:

$$
\begin{gathered}
W_{(0,0)}=\frac{1}{81}(T(3 T-10)+9)^{2}, \\
W_{(0, \pm 1)}=\frac{T}{48}(3-T)(T(3 T-10)+9), \\
W_{( \pm 1, \pm 1)}=\frac{9 T^{2}}{256}(T-3)^{2}, \\
W_{(0, \pm 3)}=\frac{T}{1296}(3 T-1)(9+T(3 T-10)), \\
W_{( \pm 1, \pm 3)}=\frac{T^{2}}{768}(T-3)(1-3 T), \\
W_{( \pm 3, \pm 3)}=\frac{T^{2}}{20736}(1-3 T)^{2} .
\end{gathered}
$$

To build equilibrium populations, the moments that are needed correspond to the momentum, pressure tensor and energy flux tensor respectively:

$$
\begin{aligned}
& M_{\alpha}=\rho u_{\alpha}, \\
& M_{\alpha \beta}=P_{\alpha \beta}^{e q}=\rho T \delta_{\alpha \beta}+\rho u_{\alpha} u_{\beta},
\end{aligned}
$$




$$
M_{\alpha \beta \gamma}=Q_{\alpha \beta \gamma}^{e q}=\rho T\left(u_{\alpha} \delta_{\beta \gamma}+u_{\beta} \delta_{\alpha \gamma}+u_{\gamma} \delta_{\alpha \beta}\right)+\rho u_{\alpha} u_{\beta} u_{\gamma} .
$$

One should note that this equilibrium is an approximation of the entropic equilibrium obtained by entropy minimization. However, both entropic equilibrium and (7) coincide to order $O\left(u^{3}\right)$, and hence we do not use the non-polynomial entropic equilibrium.

For quasi-equilibrium populations the second and third order moments are defined based on the Prandtl number. For $\operatorname{Pr} \leq \operatorname{Pr}_{\mathrm{BGK}}$ :

$$
\begin{gathered}
M_{\alpha \beta}=P_{\alpha \beta}^{*}=P_{\alpha \beta}^{e q}, \\
M_{\alpha \beta \gamma}=Q_{\alpha \beta \gamma}^{*}=Q_{\alpha \beta \gamma}-u_{\alpha}\left(P_{\beta \gamma}-P_{\beta \gamma}^{e q}\right)-u_{\beta}\left(P_{\alpha \gamma}-P_{\alpha \gamma}^{e q}\right)-u_{\gamma}\left(P_{\alpha \beta}-P_{\alpha \beta}^{e q}\right) .
\end{gathered}
$$

For $\operatorname{Pr}>\operatorname{Pr}_{\mathrm{BGK}}$ we have:

$$
\begin{gathered}
M_{\alpha \beta}=P_{\alpha \beta}^{*}=P_{\alpha \beta}, \\
M_{\alpha \beta \gamma}=Q_{\alpha \beta \gamma}^{*}=Q_{\alpha \beta \gamma}^{e q}+u_{\alpha}\left(P_{\beta \gamma}-P_{\beta \gamma}^{e q}\right)+u_{\beta}\left(P_{\alpha \gamma}-P_{\alpha \gamma}^{e q}\right)+u_{\gamma}\left(P_{\alpha \beta}-P_{\alpha \beta}^{e q}\right),
\end{gathered}
$$

where $P_{\alpha \beta}$ and $Q_{\alpha \beta \gamma}$ are the natural moments:

$$
\begin{gathered}
P_{\alpha \beta}=\sum_{i=1}^{Q} f_{i} c_{i \alpha} c_{i \beta}, \\
Q_{\alpha \beta \gamma}=\sum_{i=1}^{Q} f_{i} c_{i \alpha} c_{i \beta} c_{i \gamma} .
\end{gathered}
$$

\subsection{Boundary Conditions}

For multi-speed models the treatment of the boundary conditions is in general more delicate as compared to standard lattices. In fact, as depicted in Fig. 1, not only some populations of the nearest to the wall node are missing (black nodes in the figure), but also some populations associated with the nodes residing in the "fluid" are missing (grey nodes in the figure). For both kind of nodes, as indicated in Fig. 1 for a curved wall, the "unknown populations" are shown with dashed lines.

In this work, the approach used in [9] for the wall treatment in the case of multi-speed lattices is adopted, and is extended to complex/curved walls. In the following, the main steps used to implement the boundary conditions are presented, making a clear difference in the case of nodes belonging to the wall and nodes belonging to the "fluid".

\subsubsection{Wall Nodes Boundaries}

The first step in the wall boundary implementation is to replace the missing populations using the bounce-back scheme,

$$
f_{i}^{b b}=f_{-i}
$$


Fig. 1 Two boundary nodes for the D2Q25 lattice. Dashed arrows represent unknown populations

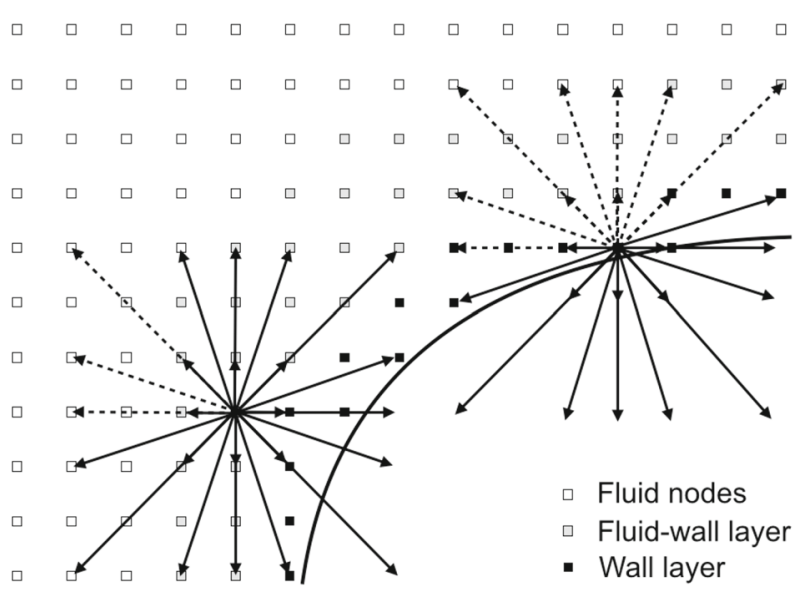

which guarantees no mass flux through the wall, and also allowing us to find the target density at the wall,

$$
\rho_{t g t}=\sum_{i \in \bar{D}} f_{i}^{b b}+\sum_{i \notin \bar{D}} f_{i}
$$

where $\bar{D}$ is the set of the missing populations. Assuming that velocity and temperature at the wall are prescribed (Dirichlet boundary conditions), we can fix target velocity, density and temperature to construct proper populations which can substitute the missing ones. In the spirit of the TMS scheme [8], the pre-collision values of the populations at the wall nodes become:

$$
f_{i} \leftarrow f_{i}+f_{i}^{\prime}\left(\rho_{t g t}, \boldsymbol{u}_{t g t}, T_{t g t}\right)-f_{i}^{\prime}\left(\rho_{l o c}, \boldsymbol{u}_{l o c}, T_{l o c}\right) .
$$

In (20), quantities indicated with the subscript $t g t$ are the target density $\rho_{t g t}$, velocity $\boldsymbol{u}_{t g t}$ and temperature $T_{t g t}$ prescribed by the Dirichlet boundary conditions for the given wall. Since for an arbitrary geometry the distance of the wall nodes from the wall is variable, linear interpolation of the far field quantities with the target quantities at the wall is performed and is employed in (20). Quantities indicated with $l o c$ are the local quantities computed using the populations at a given node, after substitution of the missing populations with approximated one at the target values:

$$
\begin{gathered}
\rho_{\text {loc }}=\sum_{i \in \bar{D}} f_{i}^{\prime}\left(\rho_{\text {tgt }}, \boldsymbol{u}_{\text {tgt }}, T_{\text {tgt }}\right)+\sum_{i \notin \bar{D}} f_{i}, \\
\boldsymbol{u}_{\text {loc }}=\frac{1}{\rho_{l o c}}\left(\sum_{i \in \bar{D}} c_{i, \alpha} f_{i}^{\prime}\left(\rho_{\text {tgt }}, \boldsymbol{u}_{\text {tgt }}, T_{\text {tgt }}\right)+\sum_{i \notin \bar{D}} c_{i, \alpha} f_{i}\right), \\
T_{l o c}=\frac{1}{D \rho_{l o c}}\left(\left(\sum_{i \in \bar{D}} c_{i}^{2} f_{i}^{\prime}\left(\rho_{\text {tgt }}, \boldsymbol{u}_{\text {tgt }}, T_{\text {tgt }}\right)+\sum_{i \notin \bar{D}} c_{i}^{2} f_{i}\right)-\rho_{l o c} \boldsymbol{u}_{l o c}^{2}\right) .
\end{gathered}
$$

It must be noted that in the TMS boundary condition of [8], the populations $f^{\prime}{ }_{i}$ are assumed to be of the form $f_{i}^{e q}$. We now modify this in the spirit of the Grad boundary conditions [14]; populations ${f^{\prime}}_{i}$ are approximated by the equilibrium populations plus a contribution of the 
non-equilibrium part estimated as:

$$
f_{i}^{\prime}=f_{i}^{e q}\left(\rho_{\text {tgt }}, \boldsymbol{u}_{t g t}, T_{t g t}\right)+f_{i}^{(1)}\left(\rho_{\text {tgt }}, \boldsymbol{u}_{t g t}, T_{t g t}, \nabla u, \nabla T\right) .
$$

The equilibrium part of the previous expression is computed according to (7), while the non-equilibrium part, is computed, as suggested in [14];

$$
f_{i}^{(1)}=\frac{W_{i}(T)}{2 T^{2}} X_{i, \alpha \beta} P_{\alpha \beta}^{(1)}+\frac{W_{i}(T)}{6 T^{3}} Y_{i, \alpha \beta \gamma} Q_{\alpha \beta \gamma}^{(1)},
$$

and where $P_{\alpha \beta}^{(1)}$ and $Q_{\alpha \beta \gamma}^{(1)}$ are the non-equilibrium pressure tensor (second order moment) and heat flux tensor (third order moment), respectively. Explicit formulation of the first order pressure and heat flux tensors is obtained by Champan-Enskog analysis and leads to:

$$
\begin{gathered}
P_{\alpha \beta}^{(1)}=-\frac{1}{\omega_{1}} \rho T\left(S_{\alpha \beta}-\frac{2}{D} \partial_{\gamma} u_{\gamma} \delta_{\alpha \beta}\right) \\
Q_{\alpha \beta \gamma}^{(1)}=-\frac{1}{\omega_{2}} \rho T\left[\partial_{\alpha} T \delta_{\beta \gamma}+\partial_{\beta} T \delta_{\alpha \gamma}+\partial_{\gamma} T \delta_{\alpha \beta}\right] \\
+u_{\alpha} P_{\beta \gamma}^{(1)}+u_{\beta} P_{\alpha \gamma}^{(1)}+u_{\gamma} P_{\alpha \beta}^{(1)}
\end{gathered}
$$

where $\omega_{1}$ and $\omega_{2}$ depend on the value of the Pr number, and $S_{\alpha \beta}$ reads

$$
S_{\alpha \beta}=\partial_{\beta} u_{\alpha}+\partial_{\alpha} u_{\beta} .
$$

The first-order pressure tensor and heat flux tensor (26) and (27) are evaluated using a second order accurate finite-difference scheme for the derivative of velocity and temperature, similarly to [16]. This procedure allows us to set to the wall nodes the correct target values of density, velocity and temperature, as well as of velocity and temperature gradients.

\subsubsection{Fluid Nodes Boundaries}

As discussed above, to complete the boundary condition implementation, also pre-collision values of missing populations belonging to the fluid nodes need to be computed. For this type of nodes the target density $\rho_{t g t}$, velocity $\boldsymbol{u}_{t g t}$ and temperature $T_{t g t}$ are chosen to be the local quantities of the previous time step, while velocity and temperature gradients are computed in the same way as for the nodes belonging to the wall. Given target quantities, the TMS algorithm described in the previous section, which includes Eqs. (20-27), is applied in exactly the same way as for the nodes nearest to the wall. It must be pointed out that with such boundary condition treatment, any kind of geometry can be simulated. Moreover, the procedure presented above for the wall boundary treatment can be applied to any type of velocity set or lattice.

\subsection{Entropic Thermal Lattice Boltzmann Formulation}

To conclude the overview on the model, extension of the higher-order TLBM to the entropic TLBM is presented in the following, based on the entropic LBM of [17]. Such an extension allows to enhance stability of sub-grid simulations. In this case, Eq. (4) is replaced by the following two equations, which depends on the Prandtl number. For $\operatorname{Pr}<\operatorname{Pr}_{B G K}$ we have:

$$
f_{i}\left(\boldsymbol{x}+\boldsymbol{c}_{i} \delta t, t+\delta t\right)-f_{i}(\boldsymbol{x}, t)=\alpha \beta_{1}\left(f_{i}^{*}-f_{i}\right)+\omega_{2}\left(f_{i}^{e q}-f_{i}^{*}\right),
$$


while for $\operatorname{Pr}>\operatorname{Pr}_{\mathrm{BGK}}$ we have:

$$
f_{i}\left(\boldsymbol{x}+\boldsymbol{c}_{i} \delta t, t+\delta t\right)-f_{i}(\boldsymbol{x}, t)=\omega_{1}\left(f_{i}^{*}-f_{i}\right)+\alpha \beta_{2}\left(f_{i}^{e q}-f_{i}^{*}\right),
$$

In the above equations, equilibrium populations $f_{i}^{e q}$ are the same as in Eq. (4), while the relaxation parameter related to the viscosity is replaced by $\alpha \beta$, where $\alpha$ is the maximal overrelaxation parameter, which is the positive root of the entropy condition:

$$
H\left(f+\alpha\left(f^{e q}-f\right)\right)=H(f) .
$$

Viscosity is now parametrized by $\beta_{1}$ or $\beta_{2}$ for Prandtl number smaller or larger than $\operatorname{Pr}_{\mathrm{BGK}}$ respectively:

$$
\mu= \begin{cases}\frac{1}{2}\left(\frac{1}{\beta_{1}}-1\right) \rho T, & \text { if } P r \leq \operatorname{Pr}_{B G K}, \\ \frac{1}{2}\left(\frac{1}{\beta_{2}}-1\right) \rho T, & \text { if } \operatorname{Pr}>\operatorname{Pr}_{B G K},\end{cases}
$$

Essentially, this formulation is based on the assumption that the entropy estimate serves to stabilize the flow only through the viscosity, without affecting the thermal conductivity. This means that application of the ELBM in the context of thermal flows is to capture the sub-grid dynamic of the velocity only.

\subsection{Thermo-Hydrodynamic Limit}

The thermo-hydrodynamic limit of the kinetic Eq. (4) was derived in [9] using the ChapmanEnskog method, assuming the Mach number is limited below the value of around $\mathrm{Ma} \simeq 0.2$ in order to keep the errors in the higher order terms as low as possible. The results consists in the Fourier-Navier-Stokes equations,

$$
\begin{gathered}
\partial_{t} \rho+\partial_{\alpha}\left(\rho u_{\alpha}\right)=0 \\
\partial_{t} u_{\alpha}+u_{\beta} \partial_{\beta} u_{\alpha}=-\frac{1}{\rho} \partial_{\alpha}(\rho T)-\frac{1}{\rho} \partial_{\beta} \Pi_{\alpha \beta}, \\
\partial_{t} T=-u_{\alpha} \partial_{\alpha} T-\frac{2}{D} T\left(\partial_{\alpha} u_{\alpha}\right)-\frac{2}{\rho D}\left(\partial_{\alpha} u_{\beta}\right) \Pi_{\alpha \beta}+\frac{2}{\rho D} \partial_{\alpha}\left(\kappa \partial_{\alpha} T\right)
\end{gathered}
$$

where the stress tensor $\Pi_{\alpha \beta}$ is defined as,

$$
\Pi_{\alpha \beta}=-\mu\left(S_{\alpha \beta}-\frac{2}{D} \partial_{\gamma} u_{\gamma} \delta_{\alpha \beta}\right),
$$

with the strain rate tensor,

$$
S_{\alpha \beta}=\partial_{\alpha} u_{\beta}+\partial_{\beta} u_{\alpha} .
$$

Expressions for viscosity and thermal conductivity as a function of the Prandtl number are given by,

$$
\mu(P r)= \begin{cases}\left(\frac{1}{\omega_{1}}-\frac{1}{2}\right) \rho T, & \text { if } \operatorname{Pr} \leq 1, \\ \left(\frac{1}{\omega_{2}}-\frac{1}{2}\right) \rho T, & \text { if } \operatorname{Pr}>1,\end{cases}
$$

and

$$
\kappa(P r)= \begin{cases}\frac{D+2}{2}\left(\frac{1}{\omega_{2}}-\frac{1}{2}\right) \rho T, & \text { if } \operatorname{Pr} \leq 1, \\ \frac{D+2}{2}\left(\frac{1}{\omega_{1}}-\frac{1}{2}\right) \rho T, & \text { if } \operatorname{Pr}>1 .\end{cases}
$$


Finally, the expression for the Prandtl number becomes:

$$
\operatorname{Pr}= \begin{cases}\frac{\left(2-\omega_{1}\right) \omega_{2}}{\left(2-\omega_{2}\right) \omega_{1},}, & \text { if } \operatorname{Pr} \leq 1, \\ \frac{\left(2-\omega_{2}\right) \omega_{1}}{\left(2-\omega_{1}\right) \omega_{2}}, & \text { if } \operatorname{Pr}>1 .\end{cases}
$$

For more detail about theoretical background, derivations and implementation the reader is referred to [9].

\section{Implementation Details}

In this work, we study the thermal flow past a circular and a square cylinder, in two dimensions, in a forced convection regime. The two-dimensional set-up is schematically drawn in Fig. 2 for the case of the two geometries considered in this work. The objects are placed at $L_{u}=5 \mathrm{D}$ downstream from the inlet, where $D$ is the characteristic size of the object, and symmetrically in the other direction. A characteristic size of the object $D=30$ lattice units have been used. With the latter as well as with larger values, the simulations results are independent from the grid size for the range of Reynolds number considered here. The width of the domain is set to $H=20 D=600$ in order to avoid influence of blockage of the flow. At the inlet (side AC in Fig. 2), all the populations are replaced with the equilibrium values that correspond to the inlet density $\rho_{0}$, velocity $U_{0}$, and temperature $T_{0}$. At the outlet (side BD in Fig. 2), missing populations are replaced by equilibrium values evaluated with the local quantities at the previous time step. In order to avoid the influence of the outlet on the bulk flow the domain is taken large enough $(L=21 D)$. On the top and bottom surfaces (sides AB and CD in Fig. 2), the free-slip boundary condition is imposed for velocity, while the adiabatic wall condition is employed for the temperature. On the object walls, the wall boundary condition described in Sect. (2.3) was applied.

The non-dimensional numbers characterizing the thermal flow are the Reynolds number,

$$
\operatorname{Re}_{\mathrm{D}}=\frac{\mathrm{U}_{0} \mathrm{D}}{v}
$$

and the Prandtl number,

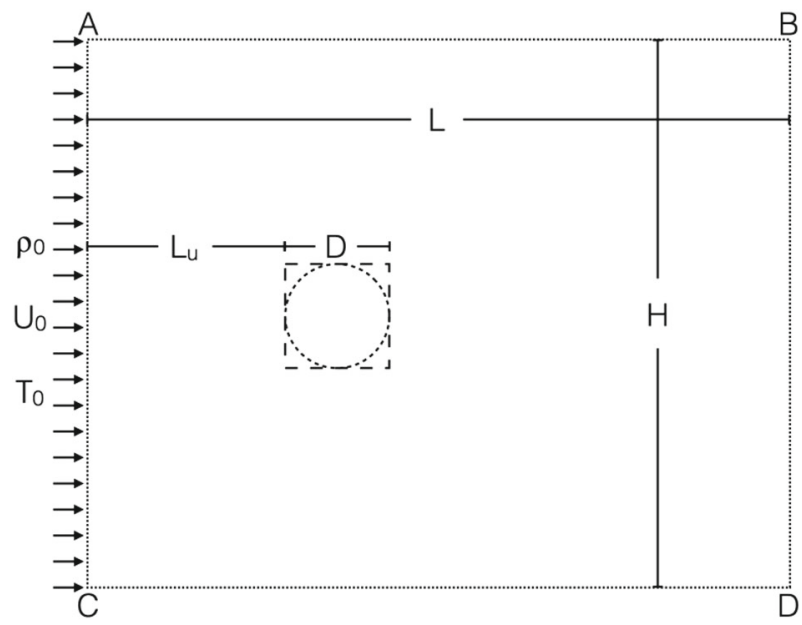

Fig. 2 Set-up for the simulation of thermal flow past a square and a circular heated obstacle 


$$
\operatorname{Pr}=\frac{\mathrm{c}_{\mathrm{p}} \mu}{\kappa} .
$$

In the present study we employ $T_{w}=T_{0}, T_{\infty}=T_{0}-\Delta T$, where $T_{0}=1-\sqrt{2 / 5}$ is the reference temperature of the lattice and $\Delta T=0.01$. The inlet velocity is set to $U_{0}=0.01$. Given different desired Reynolds numbers and Prandtl numbers, $v$ and $\kappa$ are computed and used to set the relaxations parameters. The Prandtl number is set to $\operatorname{Pr}=0.72$.

For the two geometries, the Reynolds number is varied between $10<\operatorname{Re}<200$ and $5<\operatorname{Re}<200$ for the circle and the square respectively.

\section{Numerical Validation}

The numerical validation of the present model is structured as follows: in the first part, an overview of the flow field at different Reynolds numbers and regimes is provided by means of streamlines and isotherms. Next, the structure of the flow is analyzed from a pure hydrodynamic point of view, and compared with available simulations and experimental results, analyzing separately steady and unsteady regimes for the two different geometries, and for the specified range of Reynolds numbers. At the end of the section, the thermal characteristics of the flow are exposed, focusing on the mean Nusselt number measured for the two objects and compared with available reference data.

\subsection{Flow and Isotherms Pattern}

\subsubsection{Streamlines}

The computational results for the circular cylinder are shown in Fig. 3 by means of streamlines at selected Reynolds numbers, $\operatorname{Re}=10, \operatorname{Re}=40, \operatorname{Re}=60$, and $\operatorname{Re}=200$, representing four different flow regimes. From a Reynolds of about $\operatorname{Re}=5$, the flow field behind the circular cylinder detaches from the downstream part of the cylinder wall and form a pair of symmetrical and stationary vortices, which reattaches downstream at the wake length $L_{r}$. Representative of this situation, are the streamlines depicted in Fig. 3 for the $\mathrm{Re}=10$ case. By increasing the Reynolds number, the size of the recirculation zone increases and the flow field is steady up to a Reynolds number of around $\operatorname{Re}=46$ [18]; this increase is clearly shown by the streamlines for the two Reynolds cases $\operatorname{Re}=10$ and $\operatorname{Re}=40$. From the critical $\operatorname{Re}=46$, the flow undergoes the first instability which leads to an oscillatory, periodic, two-dimensional flow: the von Kàrmàn vortex street. The instantaneous streamlines at $\mathrm{Re}=60$ in Fig. 3 represents this mode of the flow. From the critical Reynolds number $\mathrm{Re}_{3 \mathrm{D}}$ on, the flow is supposed to become three dimensional, with different evolving modes of the vortex street. There are many suggestions from experimental, numerical and analytical works for the value of $\operatorname{Re}_{3 D}$, and it is found to be in the range between $140<\operatorname{Re}_{3 \mathrm{D}}<190$ $[18,19]$.For this reason, we did not perform simulations in this work for $\operatorname{Re}>200$. However, thanks to the entropic formulation of the lattice Boltzmann equation, simulations can be run stably for Reynolds numbers up to $\operatorname{Re}=5000$ on the same grid $(D=30)$. The streamlines for the situation of $\mathrm{Re}=200$ are shown in Fig. 3 for completeness. Similarly to the circular case, streamlines of the square cylinder are shown in Fig. 4 at the same selected Reynolds numbers, $\operatorname{Re}=10, \operatorname{Re}=40, \operatorname{Re}=60$, and $\operatorname{Re}=200$, representing the same flow regimes described above.

The steady regime with a separated flow is found to be in the range $5<\operatorname{Re}<40$ [20,21] for a square two-dimensional cylinder, and it is represented in Fig. 4 by the two Reynolds 

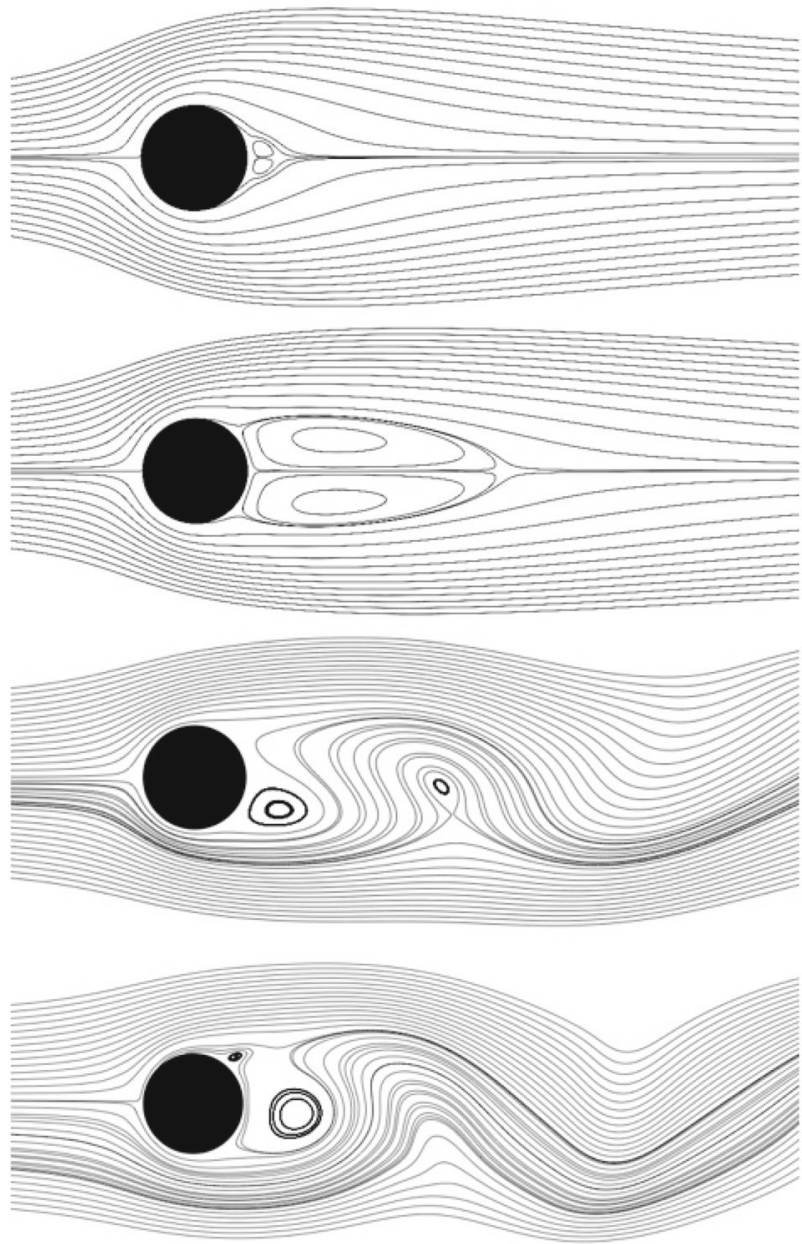

Fig. 3 Streamlines around the circular cylinder for four different Reynolds numbers. In order from the top: $\mathrm{Re}=10, \operatorname{Re}=40, \operatorname{Re}=60$, and $\operatorname{Re}=200$

$\operatorname{Re}=10$ and $\operatorname{Re}=40$. The unsteady two-dimensional regime ranges from a Reynolds of around $\operatorname{Re}=40$ to a critical Reynolds between $150<\operatorname{Re}_{3 \mathrm{D}}<175$ [20,22], and the streamlines representatives of this situation are shown in Fig. 4 for $\operatorname{Re}=60$. Same as for the cylinder, the range for the Reynolds of the simulations has been considered only until $\operatorname{Re}=200$ due to transition to a three-dimensional flow.

To conclude this sub-section, we have to stress that the streamlines for both circular and square cylinder compare qualitatively very well with the literature results $[19,21,23]$.

\subsubsection{Isotherms}

Figures 5 and 6 show the instantaneous isotherms for circular and square cylinder respectively, at the same Reynolds numbers as for the streamlines of the previous section. For both geometries, the isotherms are symmetric with respect to the downstream direction in 

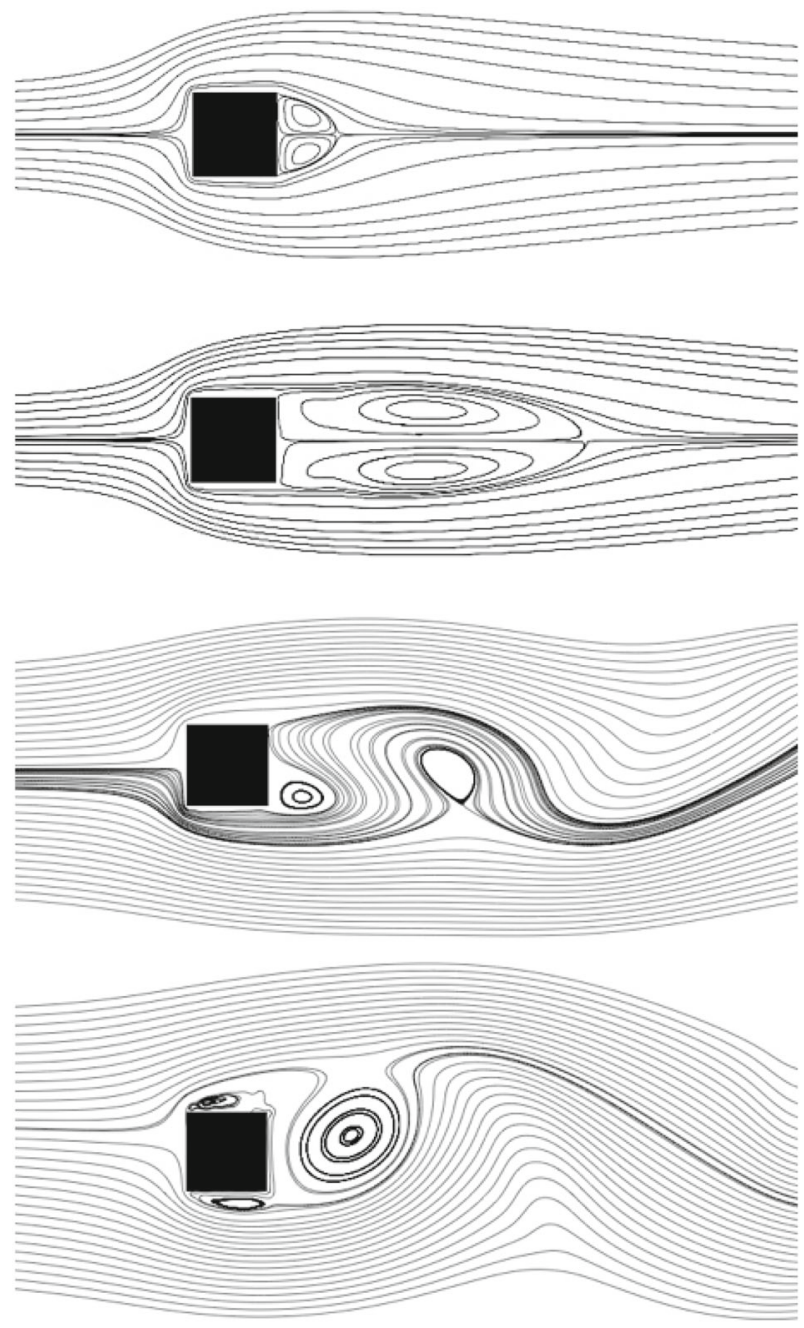

Fig. 4 Streamlines around the square cylinder for four different Reynolds numbers. In order from the top: $\mathrm{Re}=10, \operatorname{Re}=40, \operatorname{Re}=60$, and $\operatorname{Re}=200$

the steady flow regime, while in the unsteady regime the temperature is transported by the vortex street, forming hot spots downstream in the flow field. Moreover, it is possible to observe that isotherms becomes more dense near the bluff body walls as the Reynolds number increases, meaning that the gradient of temperature becomes steeper near the wall by increasing Reynolds. In general, the front surface for both geometries has the maximum clustering of isotherms indicating the maximum gradients of temperature are in this region, and therefore also the maximum local Nusselt number.

Interesting to observe is that by increasing the Reynolds number the recirculation zone increases, for both geometries, in the steady zone, and it decreases in the unsteady zone, while the crowding of isotherms in the rear part of the geometry continuously increases for both range of Reynolds for both geometries. This is mainly due to the increases of recirculating 

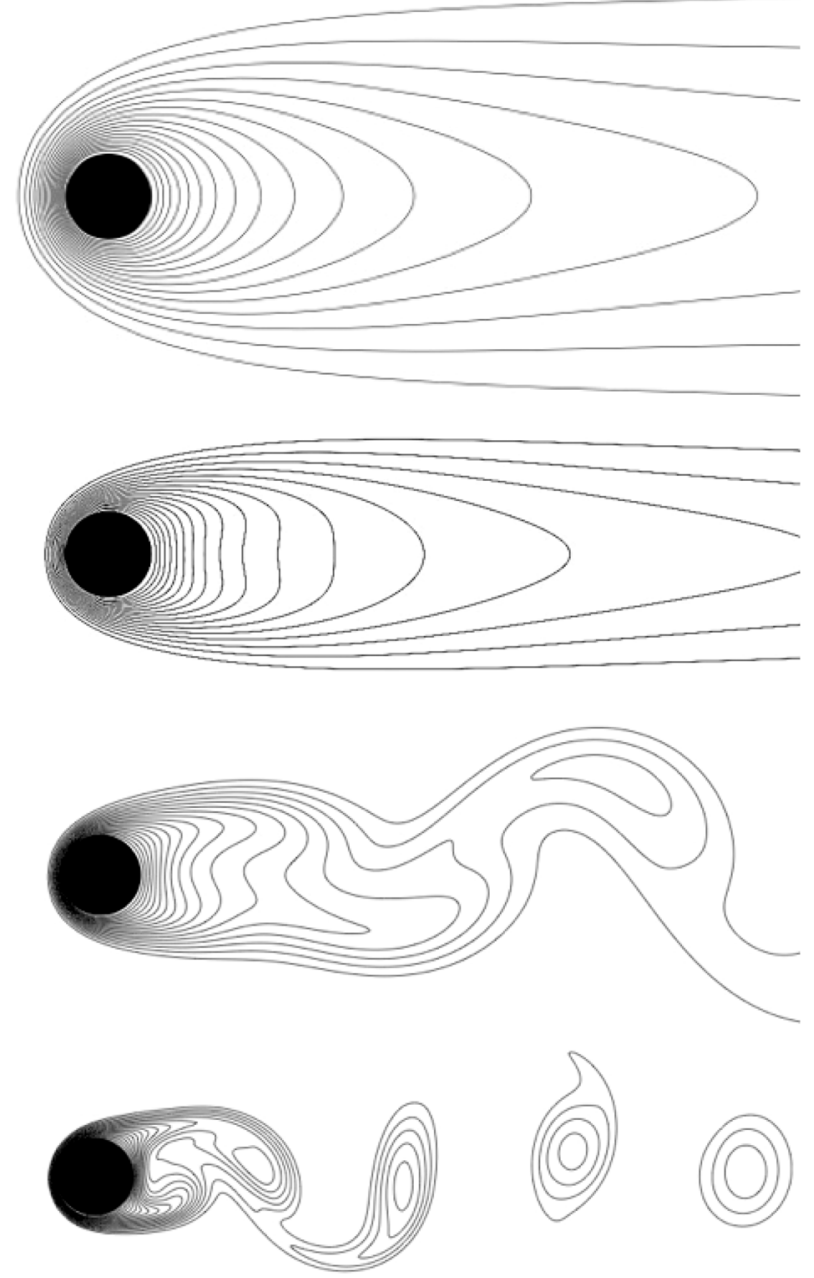

Fig. 5 Isotherms around the circular cylinder for four different Reynolds numbers. In order from the top: $\mathrm{Re}=10, \operatorname{Re}=40, \operatorname{Re}=60$, and $\operatorname{Re}=200$. Step between each isotherms is $\delta T=0.0005$

flow in the steady regime, and due to the rolling of the von Kàrmàn vortices in the unsteady periodic regime [21].

The isotherms for both circular and square cylinder compare qualitatively very well with the literature results $[21,24]$.

\subsection{Flow Structure}

As anticipated above, in the range of Reynolds numbers considered for the model validation, the flow assumes mainly two regimes: a steady and an unsteady regime. In Fig. 7, the wakebubble length behind the circular and square cylinders is plotted as function of the Reynolds number, in the steady flow regime. The wake length is the measure of the distance between the two stagnation points behind the bluff body: one attached to the solid geometry, the other in the free-stream. The present results are shown together with the reference values of $[25,26]$ for the circular cylinder, and of [21,27] for the square. 

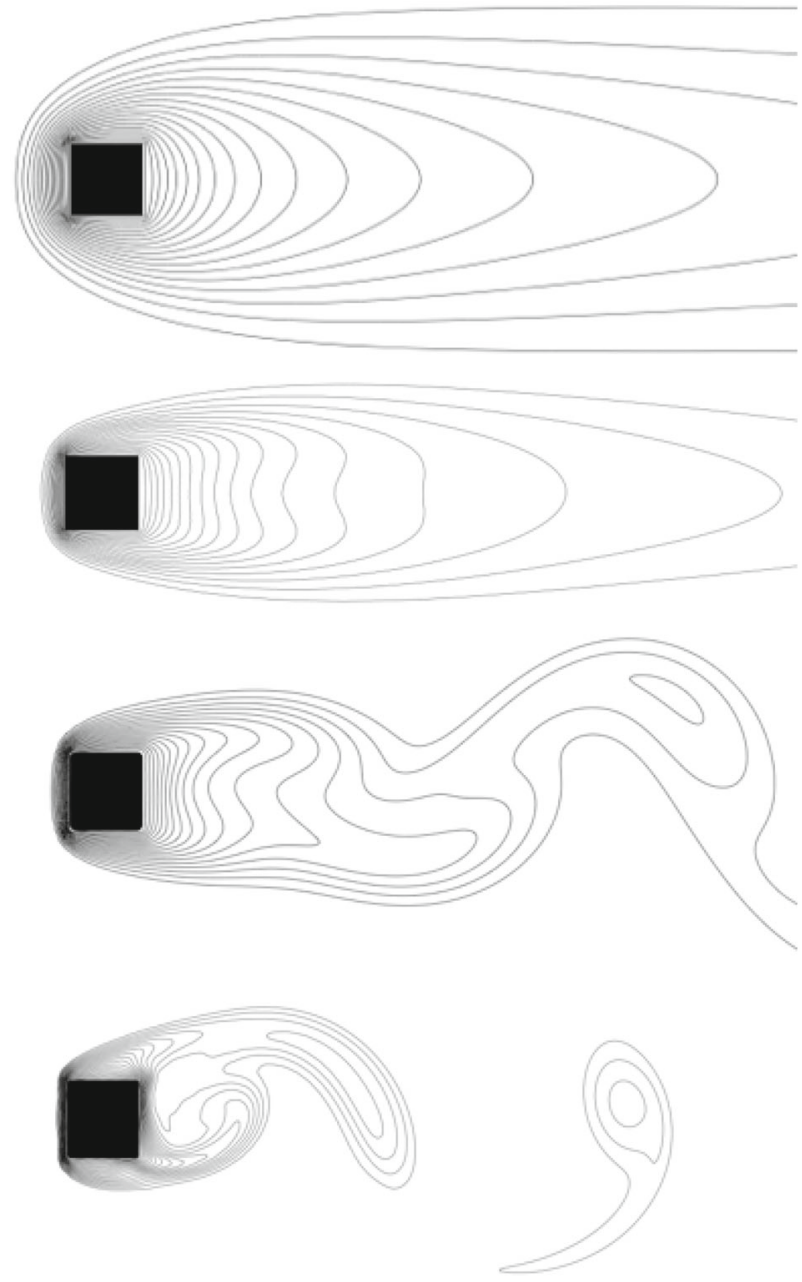

Fig. 6 Isotherms around the square cylinder for four different Reynolds numbers. In order from the top: $\mathrm{Re}=10, \operatorname{Re}=40, \operatorname{Re}=60$, and $\operatorname{Re}=200$. Step between each isotherms is $\delta T=0.0005$

In the unsteady regime, a characteristic length of the flow is the mean wake recirculation length, which consists in measuring the wake length similarly as for the steady case, but for the mean flow field averaged over many vortex shedding cycles. In Fig. 8 the computed mean wake recirculation lengths are presented for the unsteady regime, for both the circular and the square geometries, together with the reference values of [28] for the circle and of [21] for square.

Both the results for steady and unsteady regimes for both the geometries of the wake length compare very well with the references, over the entire Reynolds number range considered in the simulations.

In order to characterize the unsteady but periodic nature of the shedding flow field induced by the first instability, important parameter is the vortex shedding frequency, usually measured in terms of the Strouhal number: 


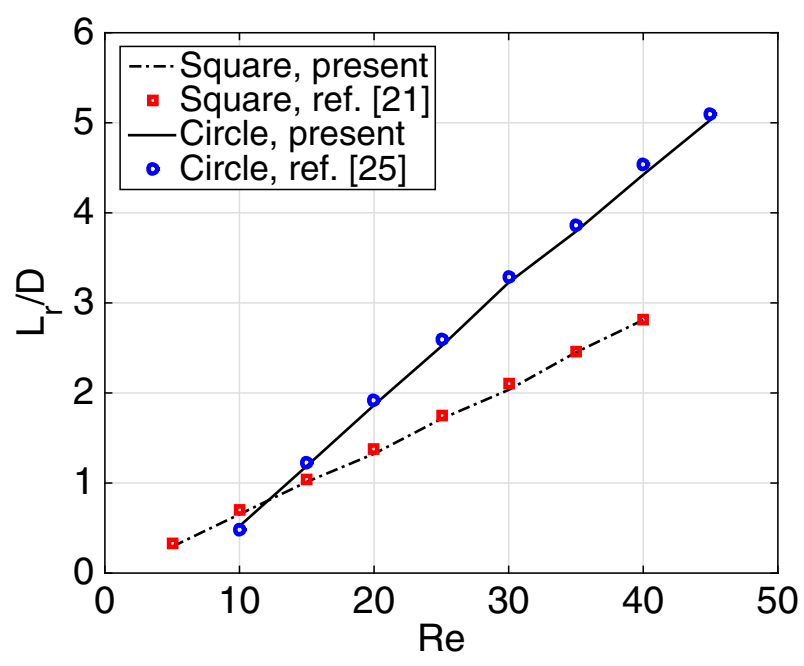

Fig. 7 Wake recirculation length for square and circular cylinder. Reference data taken from [25,26] for the circular cylinder, and from [21,27] for the square

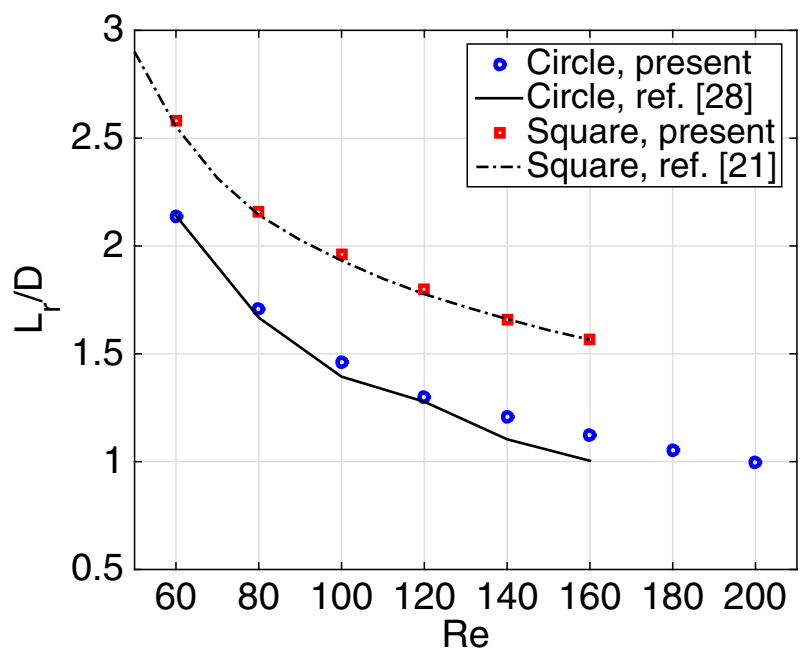

Fig. 8 Mean wake recirculation length for square and circular cylinder. Reference data taken from [28] for the circular cylinder, and from [21] for the square

$$
\mathrm{St}=\frac{\mathrm{f} \mathrm{D}}{\mathrm{U}_{\infty}}
$$

In order to compute the Strouhal number, a fast Fourier transform of the time series for the y-velocity component, $v(t)$, measured $2.5 \mathrm{D}$ downstream the solid, was performed. The characteristic frequency of the flow is extracted then from the fast Fourier transform giving the corresponding peak. The results for the Strouhal number computed for the simulations of the circular cylinder are shown in Fig. 9, and compared with the experimental results of [29,30]. 


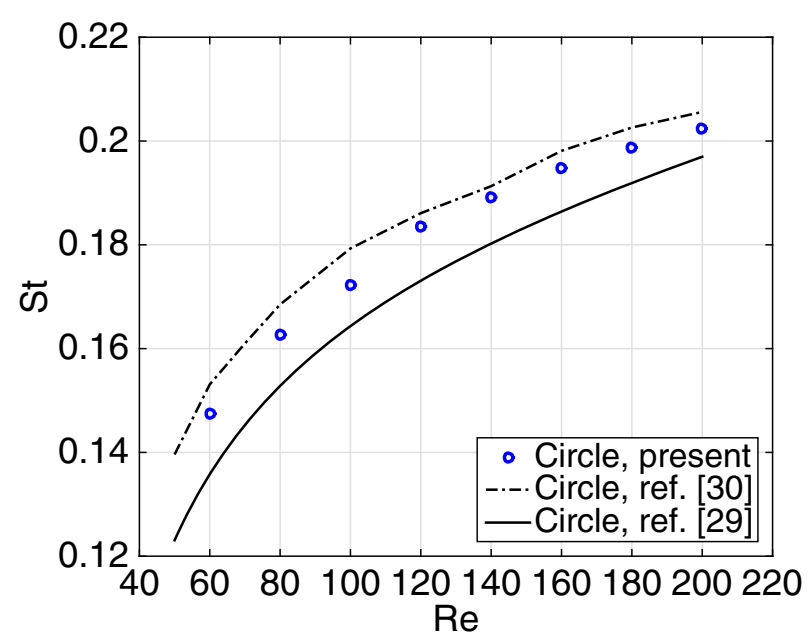

Fig. 9 Strouhal number versus Reynolds number for circular cylinder. Reference data from [29,30]

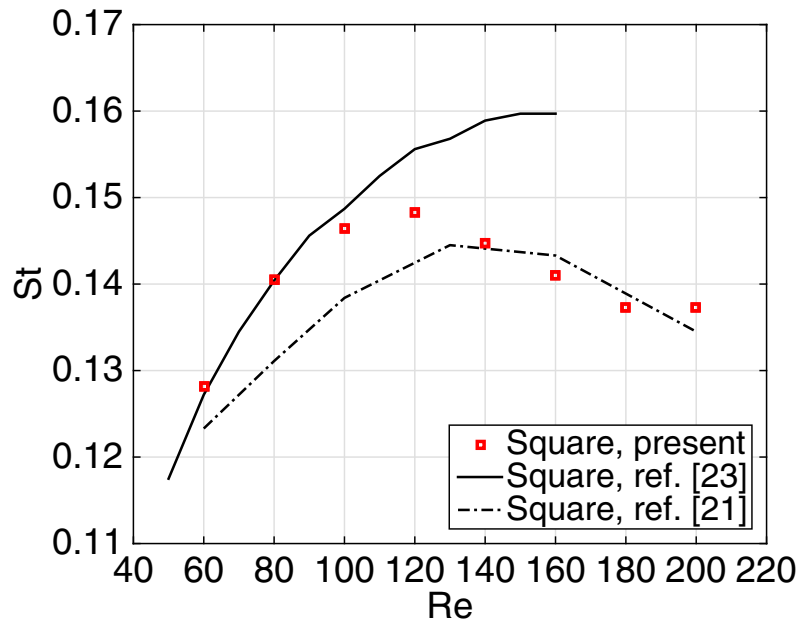

Fig. 10 Strouhal number versus Reynolds number for the square cylinder. Reference data from [21,23]

The same plot, but for the square geometry, in shown in Fig. 10, where reference data $[21,23]$ has been used for comparison.

For both geometries the Strouhal number is near or in between the references provided, even if the range of Reynolds number is quite restricted, and the sensitivity in the measure of the Strouhal number is therefore highly affected. The results provided for the wake structure and the wake shedding in the unsteady regime suggests that the model and the boundary conditions presented in this work are able to reproduce the correct characteristics of the flow from the hydrodynamic point of view.

\subsection{Flow Heat Transfer}

An estimation of the convective heat transfer between the solid and the fluid is provided by the Nusselt number. The local Nusselt number is evaluated by: 


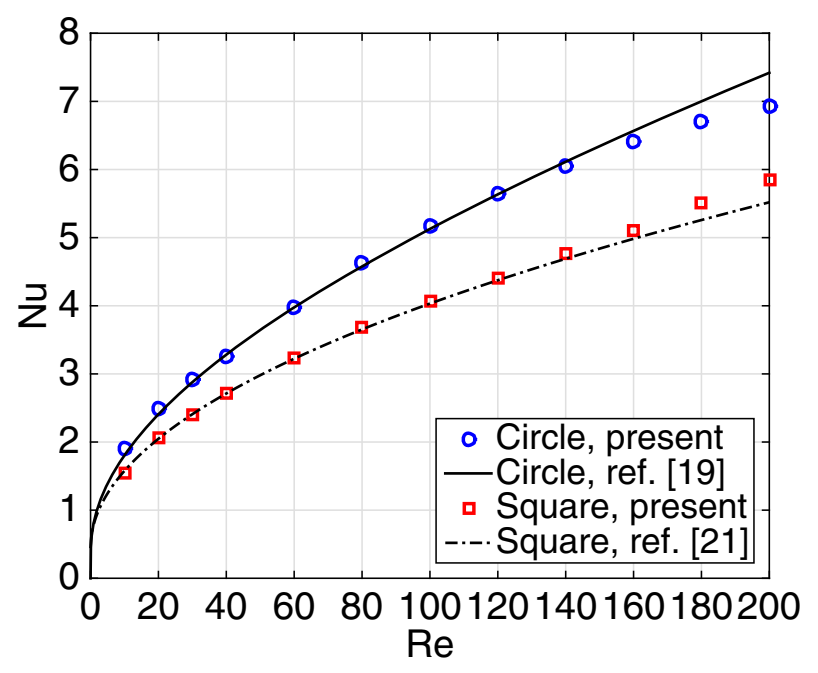

Fig. 11 Nusselt number versus Reynolds number for circular and square cylinder. Reference data from [19] for the circle and from [21] for the square

$$
\mathrm{Nu}=-\frac{\partial \theta}{\partial \mathrm{n}}
$$

where $\theta=\frac{T-T_{\infty}}{T_{w}-T_{\infty}}$ is the reduced temperature, and $n$ is the coordinated normal with respect to the wall. The average Nusselt number over the entire geometry wall can then be evaluated by:

$$
\mathrm{Nu}_{\mathrm{m}}=\frac{1}{\mathrm{~N}} \sum_{\mathrm{i}=1}^{\mathrm{N}} \mathrm{Nu}_{\mathrm{i}},
$$

where $\mathrm{Nu}_{\mathrm{i}}$ is the Nusselt number measured at the sample point $i$ of $N$ points distributed on the wall. Evaluation of the mean Nusselt number is performed on-line, in the in-house code, after advection and boundary conditions, and after locally conserved fields were computed.

The average Nusselt number for the circular and square cylinders is plotted as a function of the Reynolds number in Fig. 11, for the steady and unsteady flow regimes. The results are compared with reference [19] for the circular case and with [21] for the square.

From a Reynolds number of about $140<\operatorname{Re}<160$ the Nusselt number starts to deviate from reference values and for both geometries. This deviation could be attributed to the tendency of the flow to become three-dimensional, while the simulations are performed in two dimensions. Apart from this deviation, the results for the Nusselt number for both geometries are very promising, and suggest that the boundary conditions presented here are suitable for both thermo and hydrodynamic computations, and for complex geometries.

\section{Conclusions}

The recently introduced entropic thermal LBM [9] is extended to curved wall boundary conditions and validated for two complex geometries; a circular and a square cylinder in twodimensions, in a forced convection regime. The two objects are heated at constant temperature and the Reynolds number is varied between 5 and 200. 
In the first part of the validation of the model, streamlines and isotherms were plotted for a qualitative analysis of the thermal and hydrodynamic field, and a qualitative comparison with available reference. Next, quantitative results characterizing the wake behind the two objects, such as wake recirculation length and Strouhal number, were compared with the results of the literature. In order to validate quantitatively the thermal field, the average Nusselt number on the two geometries was computed for a range of Reynolds numbers and they were also compared with the references. In general, the results for both wake structure, vortex shedding frequency and Nusselt number measurements compare very well with both numerical and experimental literature. Both the thermal model and the boundary conditions of [9] are then a promising alternative for the simulation of forced and natural convection in complex geometries, paving the way for the simulation of 3D geometries in thermal and compressible flows with multi-speed lattices.

Acknowledgments This work was supported by European Research Council (ERC) Advanced Grant No. 291094-ELBM. Computational resources from the Swiss National Super Computing Center CSCS were provided under the Grant No. s492.

\section{References}

1. Chen, H., Kandasamy, S., Orszag, S., Shock, R., Succi, S., Yakhot, V.: Extended Boltzmann kinetic equation for turbulent flows. Science 301(5633), 633-636 (2003)

2. Succi, S.: The Lattice Boltzmann Equation for Fluid Dynamics and Beyond. Clarendon Press, Oxford (2001)

3. Mendoza, M., Karlin, I., Succi, S., Herrmann, H.: Relativistic lattice Boltzmann model with improved dissipation. Phys. Rev. D. 87(6), 065027 (2013)

4. Benzi, R., Succi, S., Vergassola, M.: The lattice Boltzmann equation: theory and applications. Phys. Rep. 222(3), 145-197 (1992)

5. Chen, H., Chen, S., Matthaeus, W.H.: Recovery of the navier-stokes equations using a lattice-gas Boltzmann method. Phys. Rev. A 45(8), R5339 (1992)

6. Chen, S., Doolen, G.D.: Lattice boltzmann method for fluid flows. Ann. Rev. Fluid Mech. 30(1), 329-364 (1998)

7. Qian, Y., d'Humières, D., Lallemand, P.: Lattice BGK models for navier-stokes equation. EPL 17(6), 479 (1992)

8. Chikatamarla, S., Karlin, I.: Entropic lattice Boltzmann method for turbulent flow simulations: boundary conditions. Phys. A 392(9), 1925-1930 (2013)

9. Frapolli, N., Chikatamarla, S.S., Karlin, I.V.: Multispeed entropic lattice Boltzmann model for thermal flows. Phys. Rev. E. 90, 043306 (2014). doi:10.1103/PhysRevE.90.043306

10. Ginzbourg, I., d'Humières, D.: Local second-order boundary methods for lattice Boltzmann models. J. Stat. Phys. 84(5-6), 927-971 (1996)

11. Ansumali, S., Karlin, I.V.: Kinetic boundary conditions in the lattice Boltzmann method. Phys. Rev. E. 66, 026311 (2002). doi:10.1103/PhysRevE.66.026311

12. Meng, J., Zhang, Y.: Diffuse reflection boundary condition for high-order lattice Boltzmann models with streaming-collision mechanism. J. Comput. Phys. 258, 601-612 (2014)

13. Latt, J., Chopard, B., Malaspinas, O., Deville, M., Michler, A.: Straight velocity boundaries in the lattice boltzmann method. Phys. Rev. E. 77(5), 056703 (2008)

14. Malaspinas, O., Chopard, B., Latt, J.: General regularized boundary condition for multi-speed lattice Boltzmann models. Comput. Fluid 49(1), 29-35 (2011)

15. Chikatamarla, S.S., Karlin, I.V.: Lattices for the lattice Boltzmann method. Phys. Rev. E. 79, 046701 (2009). doi:10.1103/PhysRevE.79.046701

16. Skordos, P.A.: Initial and boundary conditions for the lattice boltzmann method. Phys. Rev. E. 48, 48234842 (1993). doi:10.1103/PhysRevE.48.4823

17. Karlin, I., Ferrante, A., Öttinger, H.: Perfect entropy functions of the lattice Boltzmann method. EPL 47(2), 182 (1999)

18. Barkley, D., Henderson, R.D.: Three-dimensional floquet stability analysis of the wake of a circular cylinder. J. Fluid Mech. 322, 215-241 (1996) 
19. Lange, C., Durst, F., Breuer, M.: Momentum and heat transfer from cylinders in laminar crossflow at $1 e-4 \leq \operatorname{Re} \leq 200$. Int. J. Heat Mass Transf. 41(22), 3409-3430 (1998)

20. Robichaux, J., Balachandar, S., Vanka, S.: Three-dimensional floquet instability of the wake of square cylinder. Phys. Fluid 11(3), 560-578 (1999)

21. Sharma, A., Eswaran, V.: Heat and fluid flow across a square cylinder in the two-dimensional laminar flow regime. Numer. Heat Transf. A 45(3), 247-269 (2004)

22. Saha, A., Biswas, G., Muralidhar, K.: Three-dimensional study of flow past a square cylinder at low reynolds numbers. Int. J. Heat Fluid Flow 24(1), 54-66 (2003)

23. Breuer, M., Bernsdorf, J., Zeiser, T., Durst, F.: Accurate computations of the laminar flow past a square cylinder based on two different methods: lattice-Boltzmann and finite-volume. Int. J. Heat Fluid Flow 21(2), 186-196 (2000)

24. Bharti, R.P., Chhabra, R., Eswaran, V.: A numerical study of the steady forced convection heat transfer from an unconfined circular cylinder. Heat Mass Transf. 43(7), 639-648 (2007)

25. Biswas, G., Sarkar, S.: Effect of thermal buoyancy on vortex shedding past a circular cylinder in cross-flow at low reynolds numbers. Int. J. Heat Mass Transf. 52(7), 1897-1912 (2009)

26. Dennis, S., Chang, G.Z.: Numerical solutions for steady flow past a circular cylinder at reynolds numbers up to 100. J. Fluid Mech. 42(03), 471-489 (1970)

27. Chatterjee, D., Mondal, B.: Effect of thermal buoyancy on vortex shedding behind a square cylinder in cross flow at low reynolds numbers. Int. J. Heat Mass Transf. 54(25), 5262-5274 (2011)

28. Park, J., Kwon, K., Choi, H.: Numerical solutions of flow past a circular cylinder at reynolds numbers up to 160. KSME Int. J. 12(6), 1200-1205 (1998)

29. Karniadakis, G.E., Triantafyllou, G.S.: Frequency selection and asymptotic states in laminar wakes. J. Fluid Mech. 199, 441-469 (1989)

30. Williamson, C.: Oblique and parallel modes of vortex shedding in the wake of a circular cylinder at low reynolds numbers. J. Fluid Mech. 206, 579-627 (1989) 